\title{
Michael Roden (ed.): Clinical Diabetes Research; Methods and Techniques
}

Wiley, 2007

\section{Charles Roberts}

Published online: 10 April 2008

(C) Humana Press Inc. 2008

This 2007 publication is a comprehensive survey of clinical research approaches employed in type- 1 and type- 2 diabetes studies. This work is timely in view of the dramatic increases in both juvenile and adult-onset disease, and will be of special interest to physician-scientists as well as to endocrine researchers considering translational studies in metabolic disease. Dr. Rodan has assembled an impressive group of authors who have addressed a wide range of topics ranging from basic, standard clinical procedures to more cutting-edge techniques such as functional MRI. Of particular interest is the inclusion in many of the chapters of both the theoretical basis for a given technique as well as a practical description of the specific details of its use. Also useful are the contributions on study approval and informed consent and statistical issues. Each aspect addressed in this book is up to date, with many of the bibliographies covering pertinent references through 2007.
Given the wide scope, not all the contents will be completely understandable to every reader, but that is not really the intent for a reference source such as this. A work such as this will not necessarily be important for a particular investigator's own area of expertise, but will be very helpful in understanding the techniques that are important in a new area of study or those employed by collaborators. There are a few small issues, including inconsistent quality of the English translation, a minimal discussion of the application and utility of important approaches such as microarray and proteomic profiling and analyses, and the inability to cover much of the genome-wide association data on susceptibility genes that have come out more recently. These are minor shortcomings, however, that do not appreciably detract from the overall usefulness of this book for a large group of potential readers.
C. Roberts $(\bowtie)$

Oregon National Primate Research Center, Oregon Health and Science University, Portland, OR, USA

e-mail: robertsc@ohsu.edu 